\title{
An Analytical Model of the First Eigen Energy Level for MOSFETs Having Ultrathin Gate Oxides
}

\author{
B. PAVAN KUMAR YADAV and ALOKE K. DUTTA
}

\begin{abstract}
In this paper, we present an analytical model for the first eigen energy level $\left(E_{0}\right)$ of the carriers in the inversion layer in present generation MOSFETs, having ultrathin gate oxides and high substrate doping concentrations. Commonly used approaches to evaluate $E_{0}$ make either or both of the following two assumptions: one is that the barrier height at the oxide-semiconductor interface is infinite (with the consequence that the wave function at this interface is forced to zero), while the other is the triangular potential well approximation within the semiconductor (resulting in a constant electric field throughout the semiconductor, equal to the surface electric field). Obviously, both these assumptions are wrong, however, in order to correctly account for these two effects, one needs to solve Schrödinger and Poisson equations simultaneously, with the approach turning numerical and computationally intensive. In this work, we have derived a closed-form analytical expression for $E_{0}$, with due considerations for both the assumptions mentioned above. In order to account for the finite barrier height at the oxide-semiconductor interface, we have used the asymptotic approximations of the Airy function integrals to find the wave functions at the oxide and the semiconductor. Then, by applying the boundary condition at the oxidesemiconductor interface, we developed the model for $E_{0}$. With regard to the second assumption, we proposed the inclusion of a fitting parameter in the wellknown effective electric field model. The results matched very well with those obtained from Li's model. Another unique contribution of this work is to explicitly account for the finite oxide-semiconductor
\end{abstract}

Manuscript received Sep. 19, 2009; revised Jul. 9, 2010. Department of Electrical Engineering Indian Institute of Technology, Kanpur UP 208016, India

E-mail: aloke@iitk.ac.in barrier height, which none of the reported works considered.

Index Terms- MOSFET, eigen energy level, ultrathin gate oxide

\section{INTRODUCTION}

With aggressive scaling of dimensions of MOSFETs over the last decade or so, in order to keep the various short-channel effects under check, the substrate doping is continually increased, while the oxide thickness keeps on reducing. The net effect is a tremendous increase in the vertical electric field at the oxide-semiconductor interface (henceforth, referred to simply as the interface), which initiates quantum mechanical effects [1-3], causing carrier confinement within a narrow potential well close to the interface, and, consequently, giving rise to the phenomenon of energy quantization. The quantized energy levels can be obtained accurately by solving the Schrödinger and Poisson equations self-consistently [46], however, it cannot be done analytically, with the result that the solution procedure becomes numerical and computationally extremely intensive and expensive.

In [4], Janik and Majkusiak solved the set of equations self-consistently and showed that neglecting the phenomenon of the energy quantization leads to an underestimation of the threshold voltage for MOSFETs with highly doped substrates. Stern [5] came up with an approach of finding the eigen energy levels analytically by making two important assumptions: one is that of infinite potential barrier height at the oxide-semiconductor interface, while the other is the familiar triangular potential well approximation. However, rigorously, none of these two assumptions is valid.

Mudanai et al. [6] presented a comprehensive analysis of the effects of the wave function penetration into the 
oxide, by solving Schrödinger and Poisson equations self-consistently. Now, the triangular potential well approximation is invoked by the researchers, since the Schrödinger equation has an analytical solution under this condition. In [7], using this approximation, Mueller and Schulz proposed a simplified method, which accounts for the energy quantization of the inversion layer charge carriers through a self-consistent set of parameterized solutions of the Poisson and Schrödinger equations.

Ma et al. [8] showed that the results for the carrier sheet density and the surface potential using the triangular potential well approximation match with those obtained from the numerical simulations very well provided that the effective field is properly chosen. Liu et al. [9] proposed an approximate model to describe the effect of the inversion layer quantization for deep submicron MOSFETs, indicating that this effect should be considered when the device is scaled down to the sub- $0.1 \mu \mathrm{m}$ regime.

For MOS structures, to the best of our knowledge, there is no existing report in the literature regarding any explicit analytical expression of the first Eigen energy level, without invoking either of the two assumptions stated earlier in this section. In 2005, Li et al. [10] proposed a semi-empirical expression for the Eigen energy level with the coefficient and exponent terms (present in Stern's [5] eigen energy model) as fitting parameters. The values of these parameters were adjusted by fitting the results obtained from their semi-empirical model with the numerical results. Though this model is semiempirical, however, the results obtained from the model match very well with the numerical results [10]. Therefore, in this work, we used the semi-empirical model proposed by $\mathrm{Li}$ et al. [10] as the reference, with which we compared the results obtained from our proposed model.

In another empirical model, Shams et al. [11] had made the exponent (present in the model of Li et al. [10]) an empirical function of the oxide-semiconductor barrier height and the substrate doping. However, the drawback of this model is that it uses too many fitting parameters, and most of them do not have any physical justification. From the foregoing discussion, it should be apparent that there exists a need for an analytical Eigen energy model, which does not use either of the two assumptions listed earlier, and, at the same time, is sufficiently accurate.
Also, an accurate evaluation of the Eigen energy level is a must for an exact estimate of the gate tunneling current [12-16], which is so very important for today's MOSFETs.

The work has been presented in the following sequence. Section II presents the details of the development of the first Eigen energy model using the asymptotic approximations of the Airy function integrals, taking into account the finite barrier height at the oxidesemiconductor interface. The effective electric field present within the semiconductor is a required parameter for this model, for which we considered the widely used effective electric field model, however, with the inclusion of a fitting parameter, as presented in Section III. The results obtained from our model, which showed an excellent match with those predicted by the semiempirical model of Li et al. [10], are presented in Section IV. Also, the proposed model for the first Eigen energy level is completely analytical, and has only one fitting parameter. The summary and conclusion of the work is presented in Section V.

\section{EIGEN ENERGY MODEL FOR FINITE OXIDE-SEMICONDUCTOR BARRIER HEIGHT}

\section{Background}

With shrinking gate oxide thickness and increasing substrate doping concentration, penetration of the wave function into the oxide increases [6]. Accurate evaluation of the quantized eigen energy levels in the semiconductor region depends on the amount of this wave function penetration into the oxide region. Therefore, in this section, the eigen energy level is modeled without forcing the wave function to vanish at the oxide-semiconductor interface. The exact analysis results in the solution in terms of the Airy function integrals. Because the exact analytical expression for the Airy function integral cannot be formed, hence, we have invoked the approximation of the asymptotic Airy function integrals. The validity of this approximation is established, and the wave functions in the oxide and the semiconductor regions are formulated after applying proper boundary conditions at the interface. This also leads to the expression of the eigen energy level in terms of the surface electric field, as 
will be shown shortly.

The solution of the Schrödinger wave equation, assuming infinite barrier height at the interface and under the triangular potential well approximation, takes the form of the Airy function, expressed as [5]:

$$
\psi(x)=\operatorname{Ai}\left(\left(\frac{2 m_{s} \mathrm{qF}_{\mathrm{s}}}{\hbar^{2}}\right)^{1 / 3}\left[\mathrm{x}-\left(\frac{\mathrm{E}_{\mathrm{i}}}{\mathrm{qF}_{\mathrm{s}}}\right)\right]\right)
$$

where $\psi(\mathrm{x})$ is the wave function, with the position coordinate $\mathrm{x}$ defined to be positive into the substrate, with the origin at the interface, $m_{s}$ is the effective mass of electrons in the semiconductor, $\mathrm{q}$ is the coulomb charge, $F_{S}$ is the surface electric field, and $E_{i}$ is the eigen energy level, expressed as [5]

$$
\mathrm{E}_{\mathrm{i}}=\left(\frac{\hbar^{2}}{2 \mathrm{~m}_{\mathrm{s}}}\right)^{1 / 3}\left[\frac{3}{2} \pi \mathrm{qF}_{\mathrm{s}}\left(\mathrm{i}+\frac{3}{4}\right)\right]^{2 / 3}
$$

where substitution of $\mathrm{i}=0$ gives the ground state energy level $\mathrm{E}_{0}$, which is of paramount importance in our work.

In Eq. (1), the Airy function integral Ai(p), with p being any general variable, is given by [2]:

$$
\operatorname{Ai}(p)=\frac{A}{2 \pi} \int_{-\infty}^{+\infty} \exp \left[j\left(p u+\frac{u^{3}}{3}\right)\right] d u
$$

where $\mathrm{A}$ is the normalization factor, and $\mathrm{u}$ is an integration variable. Equation (3) is not solvable analytically, and the solution requires numerical integration. However, there are asymptotic approximations for the Airy function integral, which can be expressed as [3]:

$$
\operatorname{Ai}(\mathrm{p}) \approx \frac{\mathrm{c}_{1} \cos \left(\frac{2}{3}|\mathrm{p}|^{3 / 2}-\frac{\pi}{4}\right)}{\sqrt{\pi}|\mathrm{p}|^{1 / 4}} \quad(\mathrm{p}<0)
$$

and

$$
\operatorname{Ai}(p) \approx \frac{c_{2} \exp \left(-\frac{2}{3} p^{3 / 2}\right)}{2 \sqrt{\pi} p^{1 / 4}} \quad(p>0)
$$

where $\mathrm{c}_{1}$ and $\mathrm{c}_{2}$ are normalization constants. Assuming all the constants $\left(c_{1}, c_{2}\right.$, and $\left.A\right)$ to be equal to unity, a qualitative plot of the results using these asymptotic approximations along with the one obtained through numerical integration of Eq. (3) is shown in Fig.1. It is to be noted that the results obtained from the asymptotic approximations diverge for $\mathrm{p}=0$, and hence, are not good estimates of the actual Airy function in this region. However, barring this small region around $p=0$, the results of the asymptotic approximations match very well with the numerical results. Hence, the next task is to find the validity of these approximations for our work, which we describe in the next section.

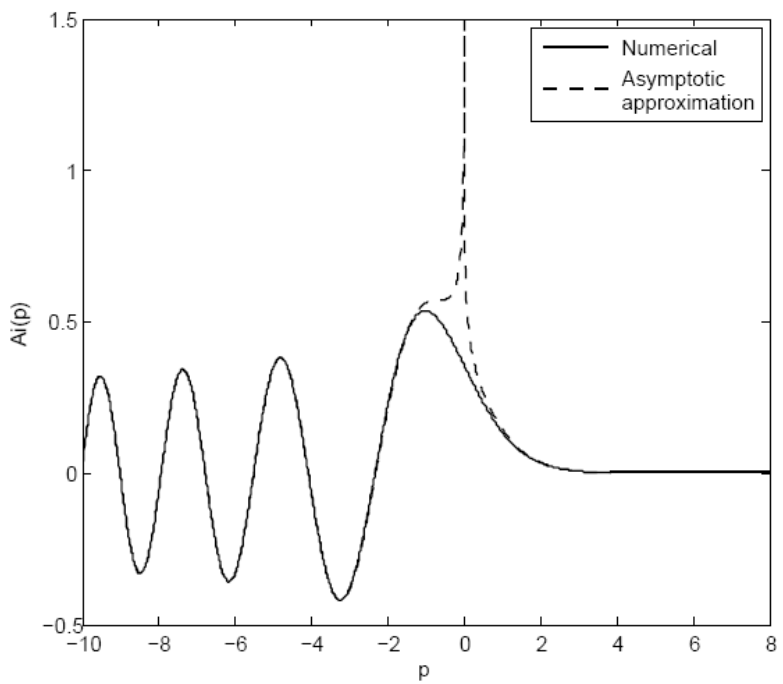

Fig. 1. Comparison of the results obtained from numerical simulation of the exact Airy function [Eq. (3)] (shown by the solid line) with those obtained using the asymptotic approximations [Eqs. (4) and (5)] (shown by the dashed line). It can be observed that except near a small region around $p=0$, the results of the asymptotic approximations match the numerical result accurately.

\section{Validity of the Approximations}

To simplify the problem at hand, we denote all the variables pertaining to the semiconductor region by suffix 1 , and those for the oxide by suffix 2 . In order to find the wave function in the semiconductor region, it is required to solve the one-dimensional Schrödinger equation, with the potential function $\phi(\mathrm{x})=\mathrm{xF}_{\mathrm{s}}$ (assuming triangular potential). The resulting wave function is identical to that given by Eq. (1), which now gets expressed as

$$
\psi_{1}(\mathrm{x})=\operatorname{Ai}\left(\mathrm{p}_{1}\right)
$$


with

$$
p_{1}=\left(\frac{2 m_{s} \mathrm{qF}_{s}}{\hbar^{2}}\right)^{1 / 3}\left[x-\left(\frac{E_{i}}{q F_{s}}\right)\right]
$$

with $\mathrm{x}=0$ corresponding to the interface. From Fig.1, it can be observed that for negative values of $p_{1}$, Eq. (4) is a very good estimate of Eq. (3) for $\mathrm{p}_{1}$ less than -1.35 (approximately). Now, if we substitute $\mathrm{x}=0$ and use the ground state eigen energy $\mathrm{E}_{0}$ predicted by Stern [Eq. (2) with $\mathrm{i}=0$ ] in Eq. (7), the result turns out to be $-(9 \pi / 8)^{2 / 3}$, which equals -2.32 , and is sufficiently less than -1.35 . Thus, the accuracy of the Airy function asymptote for $p_{1}$ $<0$ [Eq. (4)] is established. We later checked that this accuracy is maintained even when we used the result for $\mathrm{E}_{0}$ obtained from our model.

Now, for the oxide region, the one-dimensional Schrödinger equation can be solved with the potential now given by $\phi(\mathrm{x})=\phi_{\mathrm{b}}+\mathrm{xF}_{\mathrm{ox}}$, where $\phi_{\mathrm{b}}$ is the oxide-semiconductor barrier height (in $\mathrm{V}$ ), having a value roughly equal to $3.2 \mathrm{~V}$ for the $\mathrm{Si}_{-} \mathrm{SiO}_{2}$ system, and $\mathrm{F}_{\mathrm{ox}}$ is the oxide electric field. Note that in the oxide region, $\mathrm{x}$ is negative, and, at the interface, i.e., for $\mathrm{x}=0$, $\phi(\mathrm{x})=\phi_{\mathrm{b}}$, as expected. The solution again takes the form of Airy function, and can be expressed as

$$
\psi_{2}(\mathrm{x})=\operatorname{Ai}\left(\mathrm{p}_{2}\right)
$$

with

$$
\mathrm{p}_{2}=\left(\frac{2 \mathrm{~m}_{\mathrm{ox}} \mathrm{qF}_{\mathrm{ox}}}{\hbar^{2}}\right)^{1 / 3}\left[\mathrm{x}-\left(\frac{\mathrm{E}_{\mathrm{i}}-\mathrm{q} \phi_{\mathrm{b}}}{\mathrm{qF_{ \textrm {ox } }}}\right)\right]
$$

where $m_{o x}$ is the effective mass for electrons in the oxide. From Eq. (9), note that for $\mathrm{x}=0$ (i.e., at the interface), $\mathrm{p}_{2}$ is positive, since $q \phi_{b}$ is invariably greater than $E_{0}$ (because of the fact that we are considering only the ground state eigen energy level corresponding to $\mathrm{i}=0$ ). Also, from Fig.1, we observe that for positive values of $\mathrm{p}_{2}$, Eq. (5) is a very good estimate of Eq. (3) for $\mathrm{p}_{2}$ more than 2 (approximately). Again, substituting the ground state eigen energy from Stern's model [Eq. (2) with $\mathrm{i}=0$ ] in the expression for $\mathrm{p}_{2}$ [Eq. (9)], and evaluating it at the interface (i.e., at $\mathrm{x}=0$ ), we get

$$
\left.\mathrm{p}_{2}\right|_{\mathrm{x}=0}=\left(\mathrm{q} \phi_{\mathrm{b}}-\left(\frac{\hbar^{2}}{2 \mathrm{~m}_{\mathrm{s}}}\right)^{1 / 3}\left(\frac{9}{8} \pi \mathrm{qF}_{\mathrm{s}}\right)^{2 / 3}\right)\left(\frac{2 \mathrm{~m}_{\mathrm{ox}}}{\hbar^{2}}\right)^{1 / 3}\left(\mathrm{qF}_{\mathrm{ox}}\right)^{-2 / 3}
$$

where we have used the principle of continuity of electric displacement across the interface (i.e., $\varepsilon_{\mathrm{s}} \mathrm{F}_{\mathrm{s}}=\varepsilon_{\mathrm{ox}} \mathrm{F}_{\mathrm{ox}}$ ).

Equation (10) clearly shows that $\left.\mathrm{p}_{2}\right|_{\mathrm{x}=0}$ has $\mathrm{F}_{\mathrm{ox}}^{-2 / 3}$ dependence. Substitution of appropriate values in this equation predicts that $\left.\mathrm{p}_{2}\right|_{\mathrm{x}=0}$ decreases monotonically from roughly 55 (for $\mathrm{F}_{\mathrm{ox}}=500 \mathrm{kV} / \mathrm{cm}$ ) to about 6 (for $\mathrm{F}_{\mathrm{ox}}$ $=10 \mathrm{MV} / \mathrm{cm}$, which, incidentally, is larger than the breakdown field for $\mathrm{SiO}_{2}$ ). Thus, even the lowest value of $\left.\mathrm{p}_{2}\right|_{\mathrm{x}=0}$ is larger than 2 , which is the minimum required value of $\mathrm{p}_{2}$ for the results obtained from the asymptotic approximation [Eq. (5)] to match with those obtained from numerical simulations of the exact Airy function integral [Eq. (3)]. Therefore, for positive values of $\mathrm{p}_{2}$, and for practical values of the oxide field, it can be safely concluded that Eq. (5) is an excellent approximation of Eq. (3). This would be true even if the exact value of $E_{0}$ obtained from this work were substituted in Eq. (9). Thus, both the approximations have been justified. In the next section, we look at the boundary conditions.

\section{The Boundary Conditions}

Let the wave functions in the semiconductor and the oxide regions (given by $\psi_{1}(\mathrm{x})$ and $\psi_{2}(\mathrm{x})$ respectively) be denoted in the p-domain by $\phi_{1}\left(\mathrm{p}_{1}\right)$ and $\phi_{2}\left(p_{2}\right)$ respectively. By denoting the variables $p_{1}$ and $\mathrm{p}_{2}$ at $\mathrm{x}=0$ (i.e., the interface) by $\mathrm{p}_{1}^{\prime}$ and $\mathrm{p}_{2}^{\prime}$ respectively, from the continuity of the wave function across the interface, and using the expressions given by Eqs. (4) and (5), we get

$c_{2} \exp \left[-(2 / 3)\left(p_{2}^{\prime}\right)^{3 / 2}\right]=2\left(p_{2}^{\prime}\right)^{1 / 4}\left(\frac{c_{1} \cos \left[(2 / 3)\left|p_{1}^{\prime}\right|^{\prime / 2}-\pi / 4\right]}{\left|p_{1}^{\prime}\right|^{1 / 4}}\right)$

Now, with the effective mass of the carrier being different on the two sides of the interface, the second 
boundary condition, i.e., that of the continuity of the derivative of the wave function at the interface, takes the following form:

$$
\left.\frac{1}{\mathrm{~m}_{\mathrm{s}}}\left[\frac{\mathrm{d} \phi_{1}\left(\mathrm{p}_{1}\right)}{\mathrm{dx}}\right]\right|_{\mathrm{x}=0}=\left.\frac{1}{\mathrm{~m}_{\mathrm{ox}}}\left[\frac{\mathrm{d} \phi_{2}\left(\mathrm{p}_{2}\right)}{\mathrm{dx}}\right]\right|_{\mathrm{x}=0}
$$

Using Eqs. (7) and (9), Eq. (12) can be written as

$$
\left.\left[\frac{\mathrm{d} \phi_{1}\left(\mathrm{p}_{1}\right)}{\mathrm{dp} \mathrm{p}_{1}}\right]\right|_{\mathrm{x}=0}=\left.\mathrm{f}_{1}\left[\frac{\mathrm{d} \phi_{2}\left(\mathrm{p}_{2}\right)}{\mathrm{dp} \mathrm{p}_{2}}\right]\right|_{\mathrm{x}=0}
$$

where $f_{1}$ is a physical constant, and is expressed as

$$
\mathrm{f}_{1}=\left(\frac{\mathrm{m}_{\mathrm{s}}}{\mathrm{m}_{\mathrm{ox}}}\right)^{2 / 3}\left(\frac{\varepsilon_{\mathrm{s}}}{\varepsilon_{\mathrm{ox}}}\right)^{1 / 3}
$$

Now, taking the derivatives of $\phi_{1}\left(\mathrm{p}_{1}\right)$ and $\phi_{2}\left(\mathrm{p}_{2}\right)$ (expressed by Eqs. (4) and (5) respectively) with respect to $\mathrm{p}_{1}$ and $\mathrm{p}_{2}$ respectively, Eq. (13) gets expressed as

$$
\left[\frac{1}{4\left|\mathrm{p}_{1}^{\prime}\right|}+\left|\mathrm{p}_{1}^{\prime}\right|^{1 / 2} \tan \left(\frac{2}{3}\left|\mathrm{p}_{1}^{\prime}\right|^{3 / 2}-\frac{\pi}{4}\right)\right]=\mathrm{f}_{1}\left[\frac{1}{4 \mathrm{p}_{2}^{\prime}}+\left(\mathrm{p}_{2}^{\prime}\right)^{1 / 2}\right]
$$

where we have used Eq. (11).

Upon algebraic simplification of Eq. (15) and using Eq. (14), we get

$\tan \left(\frac{2}{3}\left|p_{1}^{\prime 3}\right|^{3 / 2}-\frac{\pi}{4}\right)=\left[\frac{m_{s}}{m_{o x}}\left(\frac{q \phi_{b}}{E_{i}}-1\right)\right]^{1 / 2}+\left[\left(\frac{E_{i}}{q \phi_{b}-E_{i}}\right)\left(\frac{\varepsilon_{s} m_{s}}{\varepsilon_{o x} m_{o x}}\right)-1\right] \frac{1}{4\left|p_{1}^{\prime \prime 3}\right|^{3 / 2}}$

Note that Eq. (16) shows the dependence of $E_{i}$ on only one parameter, and that is the surface electric field $F_{S}$ [through the term $p_{1}^{\prime}-$ refer to Eq. (7)]. Now, Eq. (16) can also be expressed as $\mathrm{S}=\mathrm{T}_{1}+\mathrm{T}_{2}$, where $\mathrm{S}$ is the only term on the left-hand-side of Eq. (16), while $T_{1}$ and $T_{2}$ are the first and second terms respectively in the same equation. Thus, the eigen energy $E_{i}$ can be obtained by solving this expression using numerical techniques. In the next section, we present the result of such an analysis which led to a further simplification of the expression given by Eq. (16), resulting in an analytical expression for the eigen energy level, function only of the surface electric field $\mathrm{F}_{\mathrm{s}}$.

\section{Further Simplification}

It can be observed that Eq. (16) has only one unknown variable $\mathrm{E}_{\mathrm{i}}$ for a given electric field $\mathrm{F}_{\mathrm{s}}$, however, from $\mathrm{Eq}$. (16), it should be obvious that it is not possible to solve for $E_{i}$ analytically. In order to understand the relative contributions of the terms $\mathrm{S}, \mathrm{T}_{1}$, and $\mathrm{T}_{2}$, Eq. (16) is numerically solved as a function of the oxide electric field $F_{\text {ox }}$, with the result shown in Fig. 2. In plotting these functions, $E_{i}$ is taken to be the ground state energy level $E_{0}$, obtained by substituting $i=0$ in Eq. (2). From the figure, it is obvious that the relative contribution of the term $T_{2}$ in the overall sum $\mathrm{S}$ is negligible as compared to that of $\mathrm{T}_{1}$, even for oxide fields as high as $10 \mathrm{MV} / \mathrm{cm}$. Hence, it can be prudently approximated that $S \approx T_{1}$, which yields [from Eq. (16)]:

$$
\tan \left(\frac{2}{3}\left|\mathrm{p}_{1}^{\prime}\right|^{3 / 2}-\frac{\pi}{4}\right) \approx\left[\frac{\mathrm{m}_{\mathrm{s}}}{\mathrm{m}_{\mathrm{ox}}}\left(\frac{\mathrm{q} \phi_{\mathrm{b}}}{\mathrm{E}_{\mathrm{i}}}-1\right)\right]^{1 / 2}
$$

Unfortunately, even this simplification failed to make

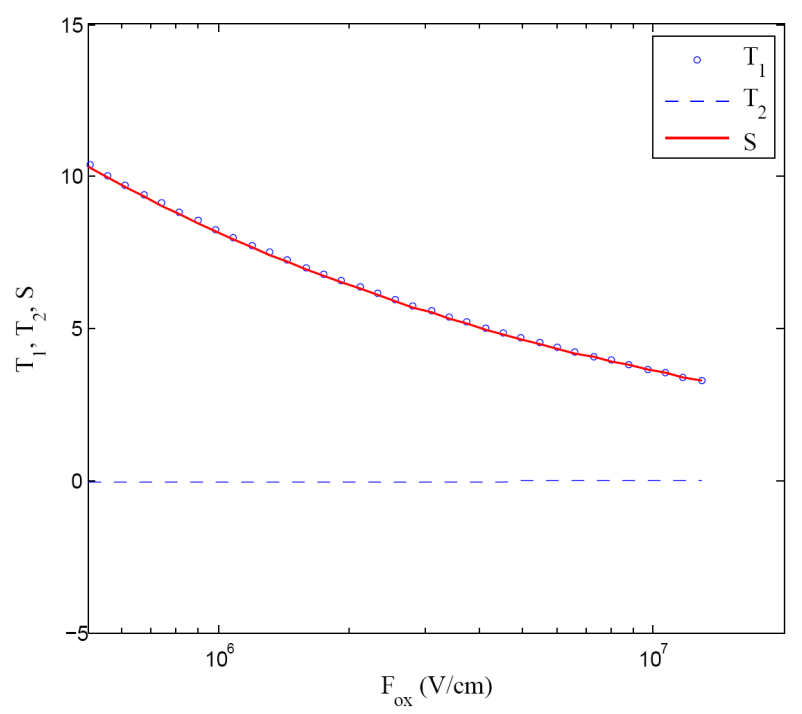

Fig. 2. The variations of the terms $S, T_{1}$, and $T_{2}$ as a function of the oxide electric field $\mathrm{F}_{\mathrm{ox}}$. Note that the contribution of the term $\mathrm{T}_{1}$ is much more dominant than that of $\mathrm{T}_{2}$ in dictating the overall sum $\mathrm{S}$, and this holds for oxide fields even beyond 10 $\mathrm{MV} / \mathrm{cm}$. 
$E_{i}$ solvable analytically from Eq. (17). Thus, we look for the next level of simplification, where we assume that the barrier height $\phi_{\mathrm{b}}$ at the interface is infinite, which leads to the first order estimate of $\mathrm{E}_{\mathrm{i}}$, as given by Eq. (2) [it can be very easily shown that Eq. (17), under the assumption that $\phi_{\mathrm{b}} \rightarrow \infty$ leads to Eq. (2)].

The rest of the procedure is algebraically slightly more tedious, however, it leads to an analytical expression of $E_{i}$, as will be shown immediately. Substituting Eq. (2) for $E_{i}$ only in the right-hand-side of Eq. (17), we get

$$
\tan \left(\frac{2}{3}\left|p_{1}^{\prime}\right|^{3 / 2}-\frac{\pi}{4}\right)=\mathrm{f}_{\mathrm{i}}\left(\mathrm{F}_{\mathrm{s}}\right)
$$

where $f_{i}\left(F_{s}\right)$ is a function of $F_{s}$ (and, of course, $\phi_{b}$, however, for a given semiconductor-insulator system, it is a constant), and is expressed as

$$
\mathrm{f}_{\mathrm{i}}\left(\mathrm{F}_{\mathrm{s}}\right)=\left[\left(\frac{\mathrm{m}_{\mathrm{s}}}{\mathrm{m}_{\mathrm{ox}}}\right)\left(\frac{\mathrm{q}_{\mathrm{b}}}{\left[\hbar^{2} /\left(2 \mathrm{~m}_{\mathrm{s}}\right)\right]^{1 / 3}\left[(3 / 2) \pi \mathrm{qF}_{\mathrm{s}}(\mathrm{i}+3 / 4)\right]^{2 / 3}}-1\right)\right]^{1 / 2}
$$

Equation (18) also implies that

$$
\left(\frac{2}{3}\left|\mathrm{p}_{1}^{\prime}\right|^{3 / 2}-\frac{\pi}{4}\right)=\mathrm{i} \pi+\tan ^{-1}\left[\mathrm{f}_{\mathrm{i}}\left(\mathrm{F}_{\mathrm{s}}\right)\right]
$$

where $\mathrm{i}=0,1,2, \cdots$, etc.

Now, by substituting $\mathrm{p}_{1}^{\prime}$ (a function of $\mathrm{E}_{\mathrm{i}}$ ) in Eq. (20) and upon further simplification, we arrive at

$$
E_{i}=\left(\frac{\hbar^{2}}{2 m_{s}}\right)^{1 / 3}\left(\frac{3 q}{2}\right)^{2 / 3}\left[\left(i+\frac{1}{4}\right) \pi+\tan ^{-1}\left[f_{i}\left(F_{s}\right)\right]\right]^{2 / 3} F_{s}^{2 / 3}
$$

Equation (21) gives an explicit analytical expression for the subband energy levels as a function of the electric field $F_{\mathrm{s}}$. The ground state energy level $\mathrm{E}_{0}$ can be obtained by substituting $i=0$ in Eq. (21), with $\mathrm{f}_{0}\left(\mathrm{~F}_{\mathrm{s}}\right)$ obtained from Eq. (19), by substituting $i=0$. Note that this expression for $\mathrm{E}_{0}$ takes into account the finite semiconduc- tor-insulator barrier height $\left(\phi_{\mathrm{b}}\right)$, which other papers in this area hitherto had not taken into account. In the next section, we develop a model for effective electric field in the semiconductor region, which should account for the triangular potential well approximation.

\section{EFFECTIVE ELECTRIC FIELD MODEL IN THE SEMICONDUCTOR REGION}

The model for $\mathrm{E}_{0}$ developed in this work so far [Eq. (21) with $\mathrm{i}=0$ ] still assumes that the electric field throughout the semiconductor is constant and is equal to the surface electric field $\mathrm{F}_{\mathrm{s}}$. However, in reality, the electric field in the semiconductor is not at all a constant rather varies from $F_{s}$ at the interface to zero deep into the bulk. It is well known that the one-dimensional Schrödinger's equation has an analytical solution when the electric field $\mathrm{F}_{\mathrm{s}}$ in the semiconductor is constant, with the potential function $\phi(\mathrm{x})$ given by $\phi(\mathrm{x})=\mathrm{xF}_{\mathrm{s}}$ (assuming triangular potential). Though there is no analytical solution for a varying electric field profile in the semiconductor region, however, attempts have been made $[4,5]$ to express this varying field by an effective field $\mathrm{F}_{\mathrm{s} \text { eff, }}$ given by

$$
\mathrm{F}_{\mathrm{s}, \mathrm{eff}}=\frac{1}{\varepsilon_{\mathrm{s}}}\left(\mathrm{Q}_{\mathrm{d}}^{\prime}+\eta \mathrm{Q}_{\mathrm{n}}^{\prime}\right)
$$

where $Q_{d}^{\prime}$ is the depletion layer charge density, $Q_{n}^{\prime}$ is the inversion layer charge density, $\varepsilon_{\mathrm{s}}$ is the permittivity of $\mathrm{Si}$, and $\eta$ is an empirical fitting parameter, which has been taken to be equal to $11 / 32$ in [5], and 0.5 in [4]. The primary goal of introducing this parameter is to account for the overestimation of the Eigen energy value, resulting from the assumption of the triangular potential well in the semiconductor region.

It has also been established in [5] that the triangular potential well (with $\eta=1$ ) is an excellent approximation in the evaluation of the Eigen energy level under the weak inversion condition, i.e., when the inversion charge density is much less than the depletion charge density. However, beyond weak inversion, the triangular potential well approximation overestimates the carrier confine- 
ment in the 2DEG at the oxide-semiconductor interface [4], and, hence, also overestimates the Eigen energy level.

In our work, we propose that using the effective electric field model $\mathrm{F}_{\mathrm{s}, \text { eff }}$ [Eq. (22)], a simple way to account for such overestimation of carrier confinement is to decrease the value of $\eta$. From extensive simulations, we have observed that the value of $\mathrm{F}_{\mathrm{s}, \mathrm{eff}}$ obtained by choosing $\eta=0.85$ makes the calculation of the Eigen energy level accurate. When we substituted $F_{s, \text { eff }}$ [Eq. (22)] (with $\eta=0.85$ ) in place of $F_{s}$ in the expression for the eigen energy level [Eq. (21) in association with Eq. (19)], it was observed that the results obtained from our model of the Eigen energy level matched very closely with that of Li et al. [10].

Now, the value of $\eta(=0.85)$ that we propose is quite higher than 0.5 and $11 / 32$ reported in [4, 5] respectively. Note that in Section II, using physics based methodology to include the effect of finite semiconductor-insulator barrier height, we have already accounted for the overestimation in $\mathrm{E}_{0}$ resulting from infinite barrier height approximation at the oxide-semiconductor interface, thus, part of the correction has already been achieved. Hence, to account for the assumption of constant electric field throughout the semiconductor region, the amount of necessary reduction in the value of $\eta$ (from its nominal value of unity) became correspondingly smaller. In the next section, we present our results.

\section{RESULTS}

In Fig. 3, we plot the values of $E_{0}$ obtained from our model as a function of the oxide electric field $\mathrm{F}_{\mathrm{ox}}$, along with the results of Stern's [5] as well as those obtained from the semi-empirical model proposed by Li et al. [10]. In plotting these results, we have used the same data as reported in $[5,10]$. Also, as a general norm, these characteristics are shown as a function of the oxide electric field $F_{o x}$ (instead of $F_{s}$ ), keeping the fact of oxide breakdown in mind. As expected, with an increase in the electric field $\mathrm{F}_{\mathrm{S}}$ (and, consequently, $\mathrm{F}_{\mathrm{ox}}$ ), the triangular potential well in the substrate becomes steeper, and the confinement of the charge carrier increases, causing an increase in the ground state eigen energy level. The same trend has been shown by the results obtained from both the models of $[5,10]$. It is interesting to note that our

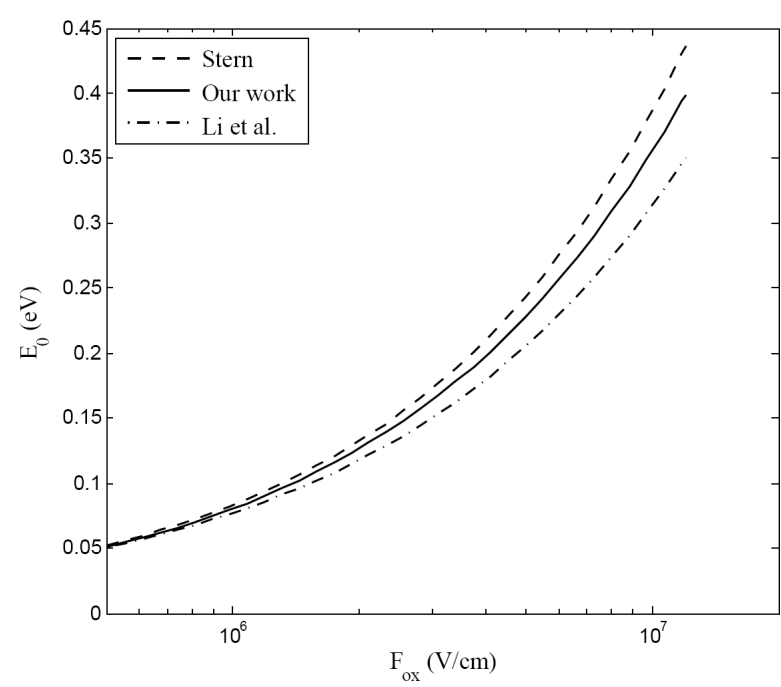

Fig.3. The ground state energy level $E_{0}$ as a function of the oxide electric field $\mathrm{F}_{\mathrm{ox}}$, obtained from our model (shown by the solid line), incorporating only the effect of finite semiconductor-insulator barrier height at the interface. The results obtained from Stern's [5] model (shown by the dashed line) as well as from the model of $\mathrm{Li}$ et al. [10] (shown by the dashdotted line) are also shown.

results are closer to that of [10], which actually showed a very good match with the results obtained from an exact numerical simulation of the coupled Schrödinger-Poisson equations [10]. Note that by taking the results reported in [10] as the reference, at oxide fields of $0.7 \mathrm{MV} / \mathrm{cm}$ and 7 $\mathrm{MV} / \mathrm{cm}$, the percent errors corresponding to the results of [5] are $6 \%$ and $20.8 \%$ respectively, while the same with respect to our model are $2.7 \%$ and $12.3 \%$ respectively, which is a non-trivial improvement.

Next, we included the model for the effective electric field $\mathrm{F}_{\mathrm{s}, \text { eff }}$ [Eq. (22)] in the original model for $\mathrm{E}_{0}$ [Eq. (21)]. The value of the coefficient $\eta$ used in the expression for $\mathrm{F}_{\mathrm{s}, \text { eff }}$ was found to equal 0.85 in order to obtain the best possible accuracy in our model of $E_{0}$. Thus, we arrive at an analytical evaluation of $E_{0}$ as a function of $\mathrm{V}_{\mathrm{GB}}$, considering both the finite barrier height at the oxide-semiconductor interface, as well as accounting for the variation of the electric field within the semiconductor region, by using the effective electric field $\mathrm{F}_{\mathrm{s}, \text { eff }}$, with a modified value of $\eta$.

In Fig. 4, we plot the variation of $\mathrm{E}_{0}$, obtained from our complete model, as a function of $\mathrm{V}_{\mathrm{GB}}$. The results obtained from the models of $[5,10]$ are also shown in the figure. The substrate doping concentration $\mathrm{N}_{\mathrm{A}}$ is taken to be $10^{17} \mathrm{~cm}^{-3}$ and the oxide thickness $t_{\mathrm{ox}}$ is equal to $2 \mathrm{~nm}$. Note the superior matching of our results (after account- 


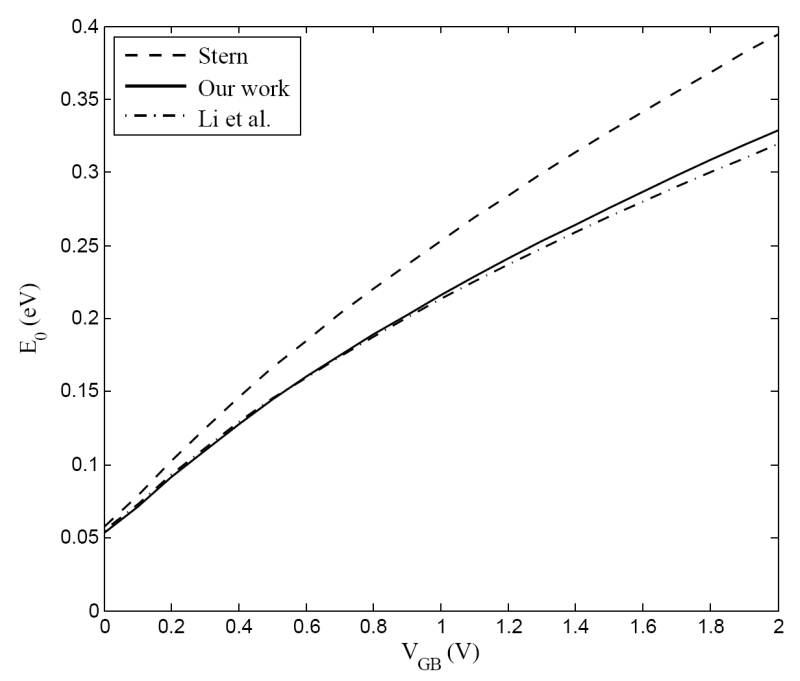

Fig. 4. $\mathrm{E}_{0}$ as a function of $\mathrm{V}_{\mathrm{GB}}$, obtained from our complete model (solid line), and the models of Stern [5] (dashed line) and $\mathrm{Li}$ et al. [10] (dot-dashed line). Note that our results emulate those of $\mathrm{Li}$ et al. $\lceil 10\rceil$ very closely.

ing for both the effects) with those predicted by the semiempirical model of [10]. In particular, by taking the result of [10] as the reference, at applied $\mathrm{V}_{\mathrm{GB}}$ of $0.5 \mathrm{~V}$ and $1.5 \mathrm{~V}$, the percent errors corresponding to the model of [5] are $14.7 \%$ and $21.5 \%$ respectively, while those of our model dropped to only $-0.3 \%$ and $2.2 \%$ respectively.

\section{V.SUMMARY AND CONCLUSIONS}

In this work, we have presented a completely analytical model for the ground state eigen energy level E0 for the quantized carriers within the inversion layer of contemporary MOSFETs, having high substrate doping and ultrathin gate oxide. It has accounted for the two important (but false) assumptions commonly used in the literature in this area, namely infinite barrier height at the oxide-semiconductor interface and the triangular potential well within the semiconductor. We took into account the effect of finite barrier height at the oxide-semiconductor interface by invoking the asymptotic approximations of the Airy function integrals, and by applying the boundary conditions at the interface, obtained the wave functions in the semiconductor as well as within the oxide - note that a nonzero wave function in the oxide is an important contribution of this work, which resulted from the barrier at the interface being finite.

From this procedure, we also obtained the expression of the ground state eigen energy level. The results matched better with those obtained from the semiempirical model of Li et al. [10] (which, in turn, match very well with the numerical simulations using the selfconsistent approach of solving Schrödinger and Poisson equations) than that of Stern [5], however, the error was still pretty high $(\sim 12 \%)$. Hence, in the next part of our work, we attempted to remove the assumption of constant electric field throughout the semiconductor region, a fallout of the triangular well approximation) by modeling it as an effective electric field, using a widely used effective electric field model, with a modified value for the parameter $\eta$. The end result was almost accurate, where the percent error in the values of E0 dropped to a meager $2 \%$ with respect to the model of $\mathrm{Li}$ et al. [10]. Also, as has been mentioned earlier, the model is purely analytical and completely based on physical principles (with prudent assumptions made and proper justifications for the same presented)

\section{REFERENCES}

[1] A. Ghatak and S. Lokanathan, "Quantum Mechanics: Theory and Applications," $5^{\text {th }}$ Ed., McMillan, 2004.

[2] L.D. Landau and E.M. Lifshitz, "Quantum Mechanics: Non-Relativistic Theory," Translated from Russian by J.B. Sykes and J.S. Bell, Course of Theoretical Physics, Vol.3, pp.491-492, 1956.

[3] Julian Schwinger, "Quantum Mechanics: Symbolism of Atomic Measurements," Edited by BertholdGeorg Englert, Springer, 2001.

[4] T. Janik and B. Majkusiak, "Analysis of the MOS transistor based on the self-consistent solution to the Schrödinger and Poisson equations and on the local mobility model," IEEE Transactions on Electron Devices, Vol.45, No.6, pp.1263-1271, 1998.

[5] F. Stern, "Self-consistent results for n-type Si inversion layers," Physical Review B, Vol.5, No.12, pp.4891-4899, 1972.

[6] S. Mudanai, L.F. Register, A.F. Tasch, and S.K. Banerjee, "Understanding the effects of wave function penetration on the inversion layer capacitance of NMOSFETs," IEEE Electron Device Letters, Vol.22, No.3, pp.145-147, 2001. 
[7] H.H. Mueller and M.J. Schulz, "Simplified method to calculate the band bending and the subband energies in MOS capacitors," IEEE Transactions on Electron Devices, Vol.44, No.9, pp.1539-1543, 1997.

[8] Y. Ma, L. Liu, Z. Yu, and Z. Li, “Thorough analysis of quantum mechanical effects on MOS structure characteristics in threshold region," Microelectronics Journal, Vol.31, No.11-12, pp.913-921, 2000.

[9] X. Liu, J. Kang, X. Guan, R. Han, and Y. Wang, "The influence of tunneling effect and inversion layer quantization effect on threshold voltage of deep submicron MOSFETs," Solid State Electronics, Vol.44, No.8, pp.1435-1439, 2000.

[10] F. Li, S. Mudanai, L.F. Register, and S.K. Banerjee, "A physically based compact gate $\mathrm{C}-\mathrm{V}$ model for ultrathin (EOT $\sqcup 1 \mathrm{~nm}$ and below) gate dielectric MOS devices," IEEE Transactions on Electron Devices, Vol.52, No.6, pp.1148-1158, 2005.

[11] I.B. Shams, K.M.M. Habib, Q.D.M. Khosru, A.N.M. Zainuddin, and A. Haque, "On the physically based compact gate $\mathrm{C}-\mathrm{V}$ model for ultrathin gate dielectric MOS devices using the modified Airy function approximation," IEEE Transactions on Electron Devices, Vol.54, No.9, pp.2566-2569, 2007.

[12] F. Rana, S. Tiwari, and D.A. Buchanan, "Selfconsistent modeling of accumulation layers and tunneling currents through very thin oxides," $A p$ plied Physics Letters, Vol.69, No.8, pp.1104-1106, 1996.

[13] J.C. Ranuárez, M.J. Deen, and C.H. Chen, "A review of gate tunneling current in MOS devices," Microelectronics Reliability, Vol.46, No.12, pp. 1939-1956, 2006.

[14] H. Wu, Y. Zhao, and M.H. White, "Quantum mechanical modeling of MOSFET gate leakage for high-k gate dielectrics," Solid State Electronics, Vol. 50. No. 6, pp. 1164-1169, 2006.

[15] R.H. Fowler and L. Nordheim, "Electron emission in intense electric fields," Proceedings of the Royal Society of London, Series A, Vol. 119, No. 781, pp. 173-181, 1928.

[16] X. Liu, J. Kang, and R. Han, "Direct tunneling current model for MOS devices with ultrathin gate oxide including quantization effect and polysilicon depletion effect," Solid State Communications, Vol.
125, No.3, pp.219-223, 2003.

[17] Y.P. Tsividis, "Operation and Modeling of the MOS Transistor," $2^{\text {nd }} E d .$, Oxford University Press, 1999.

[18] M. Shur, "Physics of Semiconductor Devices," Prentice Hall, 1995.

[19] A.K. Dutta, "Semiconductor Devices and Circuits," Oxford University Press, 2008.

[20] D. Basu and A.K. Dutta, "An explicit surfacepotential-based MOSFET model incorporating the quantum mechanical effects," Solid State Electronics, Vol.50, No.7-8, pp.1299-1309, 2006.

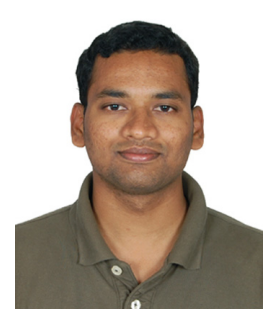

\section{B. PAVAN KUMAR YADAV was} born in Hyderabad, India on June 27, 1985. He received the B.E. degree in Electronics \& Communication Engineering from C.B.I.T, Osmania University, Hyderabad, India, in 2006 and Masters degree in Microelectronics \& VLSI from Indian Institute of Technology Kanpur, India, in 2008.

Since 2008, he has been employed with IBM Semiconductor Research and Development Center (SRDC), IBM India Pvt. Ltd, Bangalore, India, as a Staff Research Engineer, where he primarily works on CMP FILL placement, methodology and optimization, CMP Process Modeling, Mask Data Preparation, OPC (Optical Proximity Correction), ORC (Optical Rule Checking), Advanced DFM Methodologies. His research interests also include integration, characterization and modeling of nanoscale devices. 


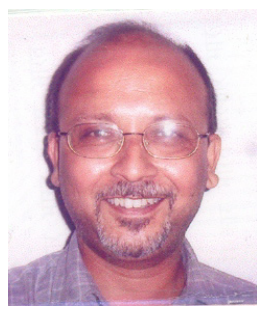

ALOKE K. DUTTA was born in Calcutta (now Kolkata), India, in 1960. He received the B.E. degree in Electrical Engineering from Jadavpur University, Calcutta (now Kolkata), India, in 1982, and the M.S. and $\mathrm{Ph}$.D. degrees in Electrical Engineering from Louisiana State University, Baton Rouge, La, USA, in 1985 and 1989 respectively. In 1990, he joined the Indian Institute of Technology, Kanpur, India, as an Assistant Professor, where he is currently a full Professor. His current research interests are focused on submicron MOSFET modeling, mixed signal low-power lowvoltage VLSI, and RF circuits. He is a member of Eta Kappa $\mathrm{Nu}$ and Phi Kappa Phi. 